\section{Schützt Screening doch nicht vor Hautkrebs-Tod?}

\author{
In der Pilotstudie SCREEN wurde der Nutzen von Hautkrebs-Reihen- \\ untersuchungen in Bezug auf die Melanommortalität wahrscheinlich \\ überschätzt. Darauf deuten detaillierte Analysen der Todesursachenstatistiken \\ aus Schleswig-Holstein und der gesamten Bundesrepublik hin.
}

Dising ie Ergebnisse der SCREEN-Studie waren ausschlaggebend für die Einführung des bundesweiten Hautkrebsscreening-Programms. Im Rahmen der Pilotstudie hatten von Juli 2003 bis Juni 2004 knapp $20 \%$ der Einwohner von Schleswig-Holstein im Alter ab 20 Jahren an einer Reihenuntersuchung auf Hautkrebs teilgenommen. Als Folge davon wurden deutlich mehr Hauttumoren diagnostiziert als in den Jahren 2001 und 2002. Fünf Jahre nach dem Screening war die Sterberate am malignen Melanom in dem Bundesland deutlich gesunken, im Vergleich zu den Jahren 1998/1999 um $47 \%$ bei den Männern und um $49 \%$ bei den Frauen. Doch jetzt melden Mediziner von der Universität Essen Zweifel an diesem potenziellen Screening-Erfolg an. Demnach könnte der Rückgang der Melanomsterblichkeit durch Ungenauigkeiten bei der Feststellung der Todesursache vorgespiegelt worden sein.

Wie aus den Todesursachenstatistiken zu erkennen ist, hat die altersstandardisierte Melanommortalität in Schleswig-
Holstein nur bis zum Jahr 2008 abgenommen. Danach ist sie wieder auf das Niveau von vor 2003 angestiegen. In absoluten Zahlen bedeutet das: 2002 starben 43 Männer und 45 Frauen am malignen Melanom, 2008 waren es nur 23 Männer und 21 Frauen. Gleichzeitig war aber ein auffälliger und vorübergehender Anstieg bei den Todesfällen mit der ICD-10-Kodierung C76-80 festzustellen: Die Zahl der bösartigen Neubildungen ungenau bezeichneter, sekundärer und nicht näher bezeichneter Lokalisationen hatte sich im selben Zeitraum von 349 bei den Männern und 356 bei den Frauen auf 480 und 434 erhöht. Ein vergleichbarer Gipfel dieser Todesursachen war in keinem anderen Bundesland zu erkennen.

Die Studienautoren vermuten daher, dass sich in Schleswig-Holstein unter den Todesfällen durch diese bösartigen Neubildungen auch einige Melanomtote finden könnten. Wären von 2007 bis 2010 pro Jahr nur 8-35 entsprechende Fehlzuordnungen bei den Frauen und 12-23 bei den Männern vorgekommen, dann wür- de das bereits den vorübergehenden Rückgang in der Melanomsterblichkeit erklären. Dafür spreche auch die Tatsache, dass die rückläufigen Zahlen bei den Melanomtoten vor allem auf eine Abnahme im nicht stationären Bereich zurückzuführen seien, wo die Todesursachenfeststellung ungenauer sei als im Krankenhaus, so die Autoren.

Anders als in Schleswig-Holstein zeigen die gesamtdeutschen Daten seit 2008, als das zweijährliche Screening-Angebot für ab 35-Jährige eingeführt wurde, keinen Rückgang der melanombedingten Todesfälle. Im Gegenteil, die altersadjustierte Mortalität hat sich von 2007 bis 2013 sogar leicht erhöht, bei Männern von 2,6 auf 3,0 und bei Frauen von 1,6 auf 1,7 pro 100.000 Personenjahre.

Fazit: Trotz dieser „enttäuschenden Ergebnisse" sind die Studienautoren nicht dafür, das Screeningprogramm zu beenden. Notwendig sei aber eine Studie, in der - anders als im SCREEN-Projekt nicht die Mortalität in der gesamten Population beobachtet, sondern die von Screening-Teilnehmern und -Nichtteilnehmern über eine längeren Zeitraum verglichen werde. Dr. Beate Schumacher

Stang A et al. Does Skin Cancer Screening Save Lives? A Detailed Analysis of Mortality Time Trends in Schleswig-Holstein and Germany. Cancer 2016; 122: 432-7

\section{Kalkbergwerk in den Extremitäten}

Eine 50-jährige Frau mit Dermatomyositis stellte sich wegen zahlreicher subkutaner Knoten an beiden Armen und Beinen vor (Abb. A). Vor 20 Jahren war die Diagnose einer Dermatomyositis gestellt worden. Damals wies die Frau Zeichen einer entzündlichen Myopathie, Gottron-Papeln und ein heliotropes und phototoxisches Exanthem auf.

Eine Behandlung mit Methotrexat und Prednisolon hatte zwar zur Remission der Myopathie geführt, die entzündlichen Hautveränderungen waren aber nie ganz zurückgegangen. In den letzten zehn Jahren waren immer mehr harte, verschiebliche, schmerzlose subkutane Knoten aufgetreten. Die Laborwerte für ionisiertes Kalzium, Phosphat, Albumin, Vitamin D und das intakte Parathormon waren normal.

Im Röntgenbild der linken Schulter (Abb. B) zeigten sich ausgedehnte Weichteilverkalkungen und eine alte Humeruskopffraktur, die man auf eine steroidinduzierte Osteoporose zurückführte. Es handelte sich um eine diffuse subkutane Kalzinose im Zusammenhang mit einer Dermatomyositis. Weichteilverkalkungen werden auch bei anderen Kollagenosen beobachtet, vor allem bei der systemischen Sklerodermie. Auch eine Behandlung mit Infliximab konnte die Tendenz zur Kalzifikation nicht aufhalten. Prof. Hermann S. FüeßI

Siddiqui NS et al. Diffuse soft-tissue calcinosis. N Engl J Med 2015; 373: 173

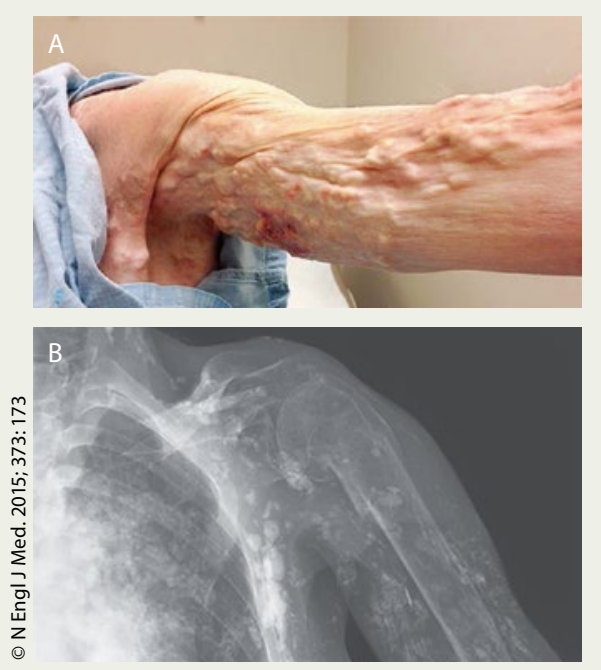

A: Subkutane Knoten am Arm; B: Weichteilverkalkungen im Röntgenbild. 